\title{
NFxB inhbitor (SC75741) -Magic stick for COVID-19
}

\author{
rimpi $\operatorname{arora}^{1}$ \\ ${ }^{1} \mathrm{IKGPTU}$
}

May 20, 2020

\begin{abstract}
The novel corona virus, previously dubbed 2019-nCoV and now officially named SARS-CoV-2 and COVID-19 has caused major outbreaks of deadly pneumonia in the 21st century has began in Wuhan, China in late 2019 and now become a destructive to global health and therefore the utmost need of the hour is to develop therapeutic candidates or vaccines against it (Zhu et al., 2020). Numerous corona viruses, first discovered in domestic poultry in the 1930s, 2002 and 2012 cause respiratory, gastrointestinal, liver, and neurologic diseases in animals. Only 7 corona viruses are known to cause disease in humans. There is an urgent need to identify specific targets to design promising therapeutic agents against severe acute respiratory syndrome coronavirus aetiological agent of coronavirus disease 2019 (COVID-19) characterised by pulmonary infection in humans. The need exists for additional treatment options addressing antiviral replication, and against SARS-CoV-2. Virus entry and replication strategies are potential targets for antiviral drug treatments. Since NF- $x \mathrm{~B}$ pathway is often targeted by viral pathogens to enhance viral replication, host cell survival and host immune evasion. Viruses may activate or suppress NFkB. (Marta et al., 2014). There have many studies on SARS-COV since 2002-2003 SARS epidemics. SARS-COV2 (COVID-19) belongs to the same family of corona viruses and shares many similarities (3), including SARS-CoV-1. Here we discuss the possible mechanisms of $\mathrm{NF} \varkappa \mathrm{B}$ inhibitor interference with the SARS-CoV-2 replication cycle.
\end{abstract}

\section{Hosted file}

$\mathrm{NF} \backslash$ selectlanguage\{greek\}\selectlanguage\{english\}B inhbitor Report.pdf available https://authorea.com/users/316953/articles/452631-nf\%CE\%BAb-inhbitor-sc75741-magicstick-for-covid-19 